\title{
Contribution of the Exact Breit Operator Plus the Electron State-Dependent Screening to the Multiplet and Fine Structure of Muon-Electron Atoms *
}

\author{
K. Rashid and B. Fricke ${ }^{\star \star}$ \\ Pakistan Institute of Nuclear Science and Technology, Nuclear Physics Division, \\ Nilore-Rawalpindi, Pakistan
}

Received January 24, 1980; revised version August 11, 1980

\begin{abstract}
We investigate for very general cases the multiplet and fine structure splitting of muonelectron atoms arising from the coupling of the electron and muon angular momenta, including the effect of the Breit operator plus the electron state-dependent screening. Although many conditions have to be fulfilled simultaneously to observe these effects, it should be possible to measure them in the $6 h-5 g$ muonic transition in the Sn region.
\end{abstract}

\section{Introduction}

With the development of the atomic Dirac-Fock (DF) method [1] it has now become possible to investigate the multiplet and fine structure features of atoms theoretically. Based on this method, which is briefly discussed in Chap. 2, we investigate here in a very general way the inclusion of the Breit operator in muon-electron atoms.

The DF calculations are performed taking into account the direct and exchange integrals of the $r^{-1}$ Coulomb interaction between the atomic particles in the self-consistent-field (SCF) procedure. The momentum of the muon $j_{\mu}$ and the momenta of the electrons $j_{e}$ couple to good angular momentum $J$. Of course, in the muon-electron interaction all exchange terms have to be omitted since electron and muon are distinguishable particles. This exclusion of the exchange integrals already gives rise to a substantial change from the usually known multiplet structure for given angular momenta. In addition to the $r^{-1}$ interaction, the Breit operator [2] is the next important contribution in the Hamiltonian of an atomic system. It consists of the magnetic and retardation part. Because retardation is non zero only in its exchange integrals, only the direct magnetic integrals appear in the muon-electron interaction. Because the Breit operator itself is only known within second order perturbation theory, we include its effect by perturbation calculation. The only case for which the

* Supported by the Pakistan-German Scientific Cooperation

$\star \star$ On leave of absence from the University of Kassel, FRG
Breit operator has been discussed in muonic atoms up to now is the system $\mu-e-$ He where its effect on the singlet and triplet state of the groundstate has been calculated [3]. In Chap. 3 we discuss the effect of the Breit operator in any possible state which may exist for a many-electron-muon system.

As a study case we have chosen lead because this nucleus is spherical and of spin zero. This allows us to avoid the angular momentum coupling between muon and nucleus and electrons and nucleus. The second reason is that most effects in muonic atoms are studied most thoroughly for this atom. In the discussion we restrict ourselves for clarity only to systems with one electron in an open shell.

Usually the inclusion of the effect of the Breit operator is called hyperfine structure, which we believe is not the most appropriate terminology for this case. We look upon this question from a general point of view and therefore treat all particles outside the nucleus equivalent as atomic cloud particles. So we have to come back to the more suitable name of the effect which is just multiplet and fine structure splitting including the magnetic interaction.

In Chap. 4 we discuss the effect of the electron statedependent screening plus the magnetic interaction. In addition, the possibility of observing these effects is investigated in detail.

\section{Method}

The general method used in these calculations is the relativistic self-consistent Dirac-Fock method. The 
details of the standard program which calculates averaged energies for electronic systems are described in $[1,4$ and 5$]$.

The total energy $E_{T}$ in a one-configuration DF calculation can be written as

$E_{T}=E_{\mathrm{av}}+\sum_{n} C_{n}^{k}(i, j ; l, m) R_{n}^{k}(i, j ; l, m)$

where $E_{\mathrm{av}}$ is the average energy of the configuration in $j-j$ coupling, which depends on the configuration and not on the particular term. $i, j, l, m$ are the indices of the one-electron wave functions with $P$ the large and $Q$ the small component. $n$ counts the total number of extra Coulomb integrals necessary to specify a given term. The Coulomb two-particle matrix elements are expressed here in terms of angular coefficients $C^{k}$ and radial integrals $R^{k}$. The radial integrals $R^{k}$ are defined as

$R^{k}(i, j ; l, m)=\iint\left[P_{i}(r) P_{j}(r)+Q_{i}(r) Q_{j}(r)\right]$

$\cdot \frac{r_{<}^{k}}{r_{>}^{k+1}}\left[P_{l}(s) P_{m}(s)+Q_{l}(s) Q_{m}(s)\right] d r d s$

with $r_{<}=\min (r, s)$ and $r_{>}=\max (r, s)$.

The direct integrals $F^{k}$ and exchange integrals $G^{k}$ are defined by

$F^{k}(i, j)=R^{k}(i, i ; j, j)$ and $G^{k}(i, j)=R^{k}(i, j ; i, j)$.

The dependence of the terms on the quantum number $J$ of the total angular momentum $\mathbf{J}$ is contained in the angular coefficients $C^{k}$. For non-closed shells these coefficients are rather complicated. They are computed by a separate program and given as input data into the Dirac-Fock program. For the $\mu-e$ interaction only the direct integrals $F^{k}$ contribute to the total energy $E_{T}$. Of course, the energy splitting of the states belonging to the possible values of total angular momentum $J$ with $\left|j_{\mu}-j_{e}\right| \leqq J \leqq\left|j_{\mu}+j_{e}\right|$ will only appear for $j_{\mu}$ and $j_{e}$ greater than $1 / 2$, because an extra Coulomb integral $F^{k}$ only exists if $k$, which is only allowed to have the values $2,4, \ldots \min \left(2 j_{\mu}-1\right.$, $2 j_{e}-1$ ), is at least equal to 2 . The Coulomb $F^{0}$ integrals cause only a common displacement. It follows that in case of $s$ or $p_{1 / 2}$ muon or electron the two possible values of the total angular momentum $J=j \pm 1 / 2$ are degenerate in energy. This degeneracy is, however, removed by the magnetic interaction in the Breit term. The Breit operator is given by the sum of the magnetic interaction $H_{B}^{M}$ and the retardation $H_{B}^{R}$

$$
\begin{aligned}
& H_{B}^{M}=-\frac{\boldsymbol{\alpha}_{1} \cdot \boldsymbol{\alpha}_{2}}{r_{12}} \\
& H_{B}^{R}=\frac{1}{2}\left\{\frac{\boldsymbol{\alpha}_{1} \cdot \boldsymbol{\alpha}_{2}}{r_{12}}-\frac{\left(\boldsymbol{\alpha}_{1} \cdot \mathbf{r}_{12}\right)\left(\boldsymbol{\alpha}_{2} \cdot \mathbf{r}_{12}\right)}{r_{12}^{3}}\right\}
\end{aligned}
$$

$H_{B}^{M+R}=-\frac{1}{2}\left\{\frac{\alpha_{1} \cdot \alpha_{2}}{r_{12}}+\frac{\left(\alpha_{1} \cdot \mathbf{r}_{12}\right) \cdot\left(\alpha_{2} \mathbf{r}_{12}\right)}{r_{12}^{3}}\right\}$

with $\boldsymbol{\alpha}$ the Dirac matrices.

In the Dirac-Fock program the contribution of the Breit term is calculated by first order perturbation theory. The first order correction to the total energy is given by

$E_{T}^{B}=E_{\mathrm{av}}^{B}+\sum_{n} C B_{n}^{k}(i, j ; l, m) R B_{n}^{k}(i, j ; l, m)$

where $E_{\mathrm{av}}^{B}$ is the average contribution of the Breit operator. $C B^{k}$ are the angular coefficients and the radial integrals $R B^{k}$ in the most general form are defined by

$R B^{k}(i, j ; l, m)=\int P_{i}(r) Q_{j}(r) \int_{0}^{r} \frac{r_{<}^{k}}{r_{>}^{k+1}} P_{l}(s) Q_{m}(s) d r d s$.

In the $\mu-e$ interaction only the direct matrix elements of the magnetic part of the Breit operator contribute to the total energy. The direct matrix elements of the retardation part of the Breit interaction are zero anyway, even for the electronelectron case. The matrix elements of the Breit operator have been investigated by Grant et al. [6] and recently by Desclaux et al. [7]. Here the angular coefficients $C B^{k}$ of the direct matrix elements are computed for the specific term and fed as input data for the calculation after the Dirac-Fock SCF procedure.

The effect of the extended nucleus is introduced by assuming a Fermi change distribution

$$
\rho(r)=\frac{\rho_{0}}{1+\exp \left(\frac{r-c}{t} 4 \ln 3\right)}
$$

with the half density radius $c$ and skin thickness parameter $t$ given by [8],c=6.63 $\mathrm{fm}, t=2.13 \mathrm{fm}$ for $\mathrm{Pb}$.

Vacuum polarization has also been included, but its effect on the term splitting is negligible, although the absolute effect is not small.

\section{Results for the Exact Breit Operator}

Energy levels and total energies are calculated for the muon-electron $\mathrm{Pb}$ atom for all configurations arising from the muon occupying the levels from $1 s$ to $4 f_{5 / 2}$ and the electron occupying the levels from $1 \mathrm{~s}$ to $2 p_{3 / 2}$, respectively (see Table 1 ). This restriction in the discussion to one electron systems is made only for clarity. (Of course, if more complicated configurations should become interesting, they also can be 
Table 1. Energy splitting in $\mathrm{eV}$ for all combinations of one muon in the levels $1 s$ to $4 f_{5 / 2}$ and one electron in the levels $1 s$ to $2 p_{3 / 2}$ coupled to good angular momenta for $\mathrm{Pb}$

\begin{tabular}{|c|c|c|c|}
\hline \multirow[t]{2}{*}{$\mu^{-}$} & \multicolumn{3}{|l|}{$e^{-}$} \\
\hline & $1 s$ & $2 p_{1 / 2}$ & $2 p_{3 / 2}$ \\
\hline $1 s$ & $\begin{array}{l}J=0 \\
16.8 \mathrm{eV} \\
J=1\end{array}$ & $\begin{array}{l}J=0 \\
0.94 \mathrm{eV} \\
J=1\end{array}$ & $\begin{array}{l}J=1 \\
0.18 \mathrm{eV} \\
J=2\end{array}$ \\
\hline $2 p_{1 / 2}$ & $\begin{array}{l}J=0 \\
5.7 \mathrm{eV} \\
J=1\end{array}$ & $\begin{array}{l}J=0 \\
0.15 \mathrm{eV} \\
J=1\end{array}$ & $\begin{array}{l}J=1 \\
0.056 \mathrm{eV} \\
J=2\end{array}$ \\
\hline $2 p_{3 / 2}$ & $\begin{array}{l}J=1 \\
21.5 \mathrm{eV} \\
J=2\end{array}$ & $\begin{array}{l}J=1 \\
1.24 \mathrm{eV} \\
J=2\end{array}$ & $\begin{array}{l}J=0 \\
0.059 \mathrm{eV} \\
J=1 \\
0.13 \mathrm{eV} \\
J=2 \\
0.18 \mathrm{eV} \\
J=3\end{array}$ \\
\hline $3 d_{3 / 2}$ & $\begin{array}{l}J=1 \\
12.8 \mathrm{eV} \\
J=2\end{array}$ & $\begin{array}{l}J=1 \\
0.74 \mathrm{eV} \\
J=2\end{array}$ & $\begin{array}{l}J=0 \\
0.083 \mathrm{eV} \\
J=1 \\
0.012 \mathrm{eV} \\
J=2 \\
0.060 \mathrm{eV} \\
J=3\end{array}$ \\
\hline $3 d_{5 / 2}$ & $\begin{array}{l}J=2 \\
27.1 \mathrm{eV} \\
J=3\end{array}$ & $\begin{array}{l}J=2 \\
1.66 \mathrm{eV} \\
J=3\end{array}$ & $\begin{array}{l}J=1 \\
0.166 \mathrm{eV} \\
J=2 \\
0.195 \mathrm{eV} \\
J=3 \\
0.160 \mathrm{eV} \\
J=4\end{array}$ \\
\hline $4 f_{5 / 2}$ & $\begin{array}{l}J=2 \\
18.8 \mathrm{eV} \\
J=3\end{array}$ & $\begin{array}{l}J=2 \\
1.18 \mathrm{eV} \\
J=3\end{array}$ & $\begin{array}{l}J=1 \\
0.24 \mathrm{eV} \\
J=2 \\
0.207 \mathrm{eV} \\
J=3,4\end{array}$ \\
\hline
\end{tabular}

calculated with this program, including the full Breit interaction between the electrons.)

As long as at least one of the particles electron or muon has an angular momentum of $j=1 / 2$, which are the $s_{1 / 2}$ and $p_{1 / 2}$ levels, the energy splitting within the same multiplett in these cases is entirely due to magnetic interaction between the muon and the electron (see Table 1).

The radial extension of the muon wavefunction is very much inside the electronic wavefunction. The overlap of both wavefunctions therefore increases for larger principal quantum numbers of the muon and decreases for larger principal quantum numbers of the electron. Therefore the radial integrals $F^{k}$ or $R B^{k}$ are largest for the electron in the $1 \mathrm{~s}$ state and the muon in a very high state. This already shows that the Coulomb splitting due to additional $F^{k}$ integrals will not be large because the electron has to occupy

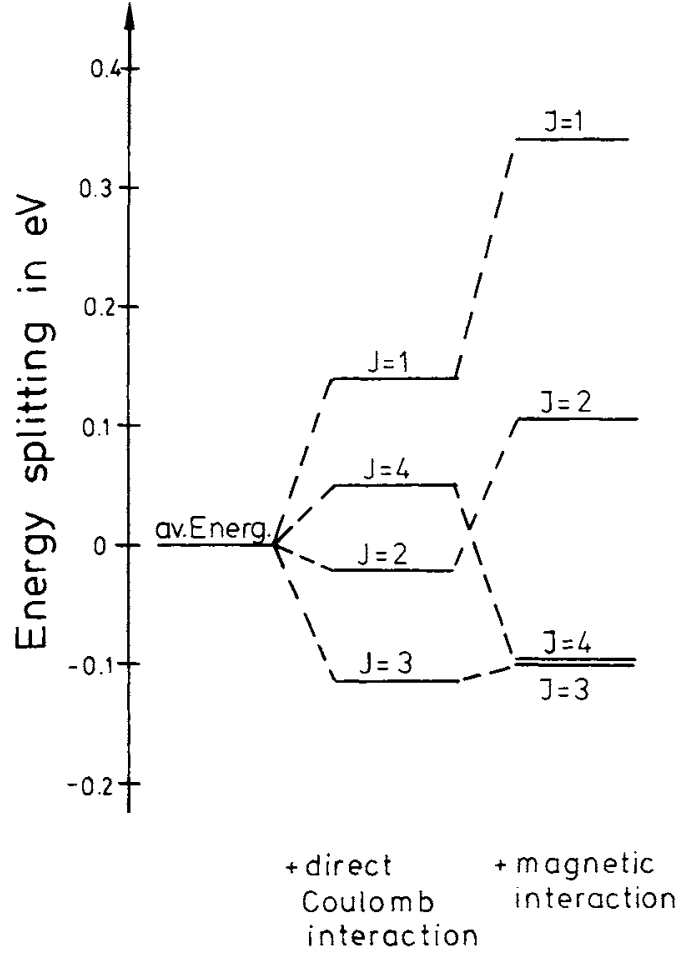

Fig. 1. The term splitting of an atomic system with one muon in the $4 f_{5 / 2}$ state and an electron in the $2 p_{3 / 2}$ state in $\mathrm{Pb}$. The level splitting due to the inclusion of the additional $F^{k}$ integrals is drastically changed after the inclusion of the direct magnetic interaction

at least the $2 p_{3 / 2}$ level or a higher one. As one can see in Table 1, the total splitting of the different terms for such cases is in the order of $10 \mathrm{eV}$ and less. On the other hand, the results in Table 1 show that the magnetic splitting of the terms where one $1 \mathrm{~s}$ electron is coupled to a muon in the $3 d_{3 / 2}$ state is up to $27 \mathrm{eV}$. Again the effect is an order of magnitude smaller if a $2 p_{1 / 2}$ electron is involved instead of a $1 s$ electron. On the other hand, the effect increases for higher muonic states.

In Fig. 1 we discuss the magnitude of the various influences on the term splitting in case of an electron in the $2 p_{3 / 2}$ state and the muon in the $4 f_{5 / 2}$ state, because here $F^{k}$ as well as $R B^{k}$ integrals contribute to the level splitting. Although the total effect in this case is very small we have chosen this case as the simplest, most general example. We find that the energy intervals between $J=1$ and $J=2$ due to Coulomb interaction is $0.15 \mathrm{eV}$. The level $J=4$ is pushed up midway between the levels $J=1$ and $J=2$. The additional magnetic splitting has a systematic trend. The $J=1$ level gets a large positive contribution which is already smaller for the $J=2$ level and nearly zero for $J=3$. The $J=4$ level has a strong negative magnetic contribution. 

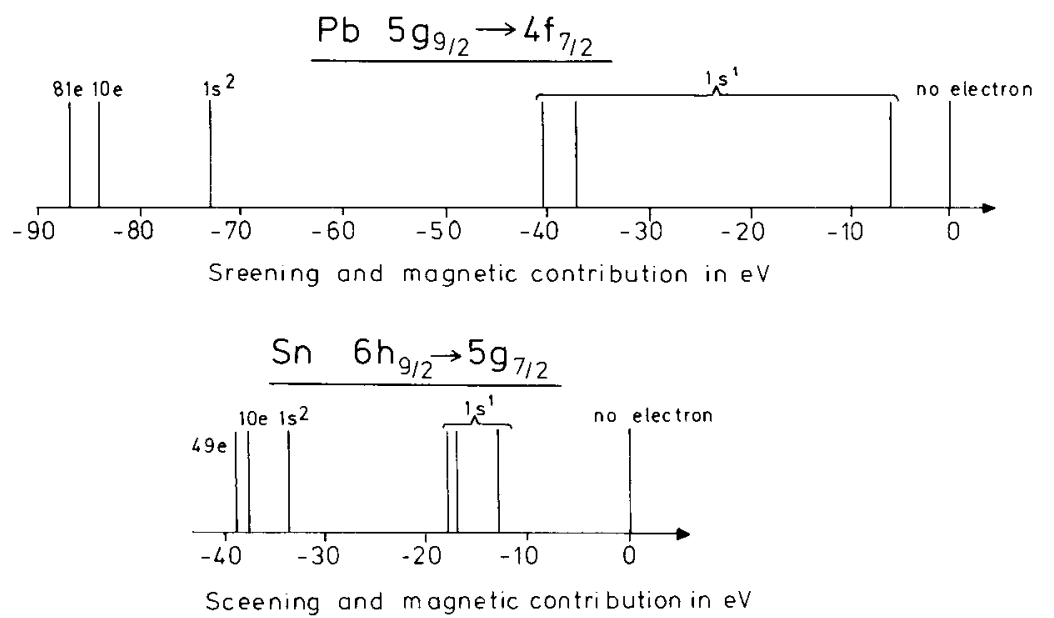

Fig. 2. Line position for muonic $5 g_{9 / 2}-4 f_{7 / 2}$ and $6 h_{9 / 2}-5 g_{7 / 2}$ transitions in $\mathrm{Pb}$ and $\mathrm{Sn}$, respectively, relative to the unscreened value. The lowest energetic groups in both cases are the transitions in the presence of the $1 s^{2}$ and more electrons. The splitting of the $1 s^{1}$ group of lines is due to the magnetic interaction. In $\mathrm{Sn}$ this group is expected to carry about $7 \%$ of the intensity

This results in closeness of the levels $J=3$ and $J=4$ for the total energy of this state. This trend is also found for the other configurations considered here. These calculations show that here in $\mathrm{Pb}$ the splitting due to Coulomb interaction and magnetic interaction is of the same order of magnitude.

\section{Inclusion of the Electron State-Dependent Screening and Discussion of a Possible Observation}

The fact that the electron screening contribution is large and becomes even larger for the higher transitions has drawn a lot of attention. Therefore, in a number of papers [9-13] this effect was calculated and the dependence on the number of electrons and sublevels was studied. Due to the radial distribution of the electrons about $85 \%$ of the screening originates from the two $1 \mathrm{~s}$ electrons as compared with the total screening of all 81 electrons in $\mathrm{Pb}$. An analysis has shown that in reality nearly always full inner shells $[11,14-16]$ have to be assumed when the muon transition occurs. This is well understood since the mean lifetime of an $1 s$ vacancy in $\mathrm{Pb}$ is of the order of $10^{-17} \mathrm{~s}$, due to the refilling of $n p$ electrons [17], whereas the radiative muon transition time for the 5-4 and 6-5 transition is at least slower by a factor of 5 and 10, respectively [18]. For higher transitions these factors are even larger. In addition, the muonic Auger transition, the process, whereby an inner electron will be ejected, has only a probability of about $2 \%$ and $8 \%$ for the transitions $6-5$ and $7-6$, respectively, as compared with radiative transition probabilities [18]. These small probabilities to generate an $1 s$ hole and its short lifetime reduce the number of 5-4 muonic transitions in the presence of an $1 s$ hole to less than $1 \%$. This number would increase to greater values if the number of $L$ electrons is smaller than 8 because of the Auger effect, so that the filling of the $1 s$ hole is not as rapid as is given in the tables [17]. On the other hand the electronic $K-\mathrm{X}$ rays in the presence of a muon with an effective charge $(Z-1)$ have been well observed [14]. We may therefore ask the question whether this effect is observable in heavier $Z$ systems. Before an answer to this question can be obtained, many conditions have to be fulfilled at the same time, namely

(a) the absolute effect of the magnetic splitting has to be large enough to be measurable. Because of its strong $Z$ dependence $Z$ should be as large as possible;

(b) the small magnitude of the effect needs a measurement with a crystal spectrometer which means that the transition energy should be less than $100 \mathrm{keV}$;

(c) the transition which is being observed should at most be radiative, whereas

(d) the preceding transition should mainly be dominated by muonic Auger transitions which will generate the $1 s$ hole, and

(e) this Auger transition has to have a transition energy which allows the creation of an $1 s$ hole energetically;

(f) the total $K$-decay rate of the $1 s$ hole has to be smaller or within the same order of magnitude as compared to the muon transition rate.

The six conditions have to be fulfilled at the same time, and this is difficult to satisfy. Nevertheless, we have found that in the $Z=50$ region this effect might be observable. For example for Tin $(Z=50)$ 
(a) the absolute effect of magnetic splitting of a $6 \mathrm{~h}$ $-5 \mathrm{~g}$ transition in the presence of an $1 \mathrm{~s}$ electron hole is in the order of $6 \mathrm{eV}$;

(b) this splitting may be measurable because this transition has an energy of about $85 \mathrm{keV}$;

(c) this transition is $80 \%$ radiative [18], whereas

(d) about $50 \%$ of the transitions which fill the $6 h$ level are Auger transitions [18].

Thus one can expect that in nearly all of these Auger transitions an $1 s$ hole is being produced because

(e) the preceding $7 i-6 h$ transition has an energy of about $51 \mathrm{keV}$, so that the $1 \mathrm{~s}$ electron with a binding energy of about $30 \mathrm{keV}$ can be ejected;

(f) because the total $K$-decay rate for the $1 \mathrm{~s}$ hole [17] is about $1.2 \cdot 10^{+16} \mathrm{~s}^{-1}$ and the muon transition rate in the $6 h-5 \mathrm{~g}$ transition $[12]$ is $2.5 \cdot 10^{10} \mathrm{~s}^{-1}$, one can expect that in about $7 \%$ of all cases the transition occurs in the presence of an $1 \mathrm{~s}$ hole.

We have calculated the energies for the muonic transitions $6 h-5 g$ in $\mathrm{Sn}$ as well as $5 g-4 f$ in $\mathrm{Pb}$ for comparison in the presence of various electronic configurations (Fig. 2). Because the relative positions of the lines are not affected by vacuum polarization, self-energy or recoil effects, only the effect of screening and magnetic splitting will manifest themselves in such a measurement. In Fig. 2 three groups of lines can be seen.

The lowest energetic group are transitions in the presence of at least two $1 \mathrm{~s}$ electrons or more. Because of the small additional screening of all outer electrons there is not much difference to the $1 s^{2}$ screening. The middle group are the transitions discussed above which are effected by the $1 s$ screening plus the magnetic splitting. Additional outer electrons may give some additional structure only a few eV apart. The highest energetic line is the very unreasonable case when both $1 \mathrm{~s}$ electrons are ionized. The discussion above has shown that the middle group of lines in $\mathrm{Sn}$ should be emitted with about $7 \%$ intensity compared to the low energetic group, whereas in $\mathrm{Pb}$ only the lowest energetic group will be measured.

The observation of these lines in Sn would give three important informations:

(1) Knowledge of the electronic structure during the muon cascade.
(2) The relative intensities of the various groups are an experimental check of the muon Auger and radiation rates, as well as the $K$-X-ray emission rate.

(3) The energetic analysis of the observed lines will give information on the magnetic interaction of the two non-identical particles muon and electron, which is of general interest.

We would like to express our gratitude to Dr. Jean-Paul Desclaux for supplying us with the additional magnetic integrals. The financial support within the Pakistan-German Scientific Cooperation and the help from PAEC, PINSTECH and $\mathrm{KfK}$ is greatly acknowledged. One of us (B.F.) would like to thank PINSTECH and PAEC for their great hospitality during his stay in Pakistan.

\section{References}

1. Desclaux, J.P.: Comput. Phys. Commun. 9, 31 (1975)

2. Breit, G.: Pnys. Rev. 35, 1447 (1930) and 37, 51 (1931)

3. Huang, K.N., Hughes, V.W.: Phys. Rev. A 20, 706 (1979)

4. Desclaux, J.P., Mayers, D.F., O'Brien, F.: J. Phys. B4, 631 (1971)

5. Mallow, J.V., Desclaux, J.P., Freeman, A.J.: Phys. Rev. A17, 1804 (1978)

6. Grant, I.P., Pyper, N.C.: J. Phys. B9, 761 (1976)

7. Desclaux, J.P., Cheng, K.T., Kim, Y.K.: J. Phys. (to be published)

8. Landolt-Börnstein, Zahlenwerte und Funktionen. Band 2. Hellwege, K.H., (ed.), Bd.2. Berlin, Heidelberg, New York: Springer 1967

9. Fricke, B.: Nuovo Cimento Lett. 2, 859 (1969)

10. Vogel, P.: Phys. Rev. A7, 63 (1973)

11. Vogel, P.: Phys. Rev. A8, 2292 (1973)

12. Mallow, J.V., Desclaux, J.P., Freeman, A.J.: Phys. Rev. A17, 1804 (1978)

13. Egidy, T. von, Desclaux, J.P.: Z. Physik A 288, 23 (1978)

14. Fromm, W.D., Gansorig, Dz., Krogulski, T., Ortlepp, H.G., Polikanov, S.M., Sabirov, B.M., Schmidt, U., Arlt, R., Engfer, R., Schneuwly, H.: Phys. Lett. B55, 377 (1975)

15. Vuilleumir, J.L., Dey, W., Engfer, R., Schneuwly, H., Walter, H.K., Zehnder, A.: Z. Physik A 278, 109 (1976)

16. Vogel, P., Zehnder, A., Carter, A.L., Dixit, M.S., Hincks, E.P., Kessler, D., Wadden, J.S., Hargrove, C.K., McKee, R.J., Mes, H., Anderson, H.L.: Phys. Rev. A 15, 76 (1977)

17. Bambynek, W., Craseman, B., Fink, R.W., Freund, H.U., Mark, H., Swift, C.D., Price, R.E., Venugopala Rao, P.: Rev. Mod. Phys. 44, 716 (1972)

18. Devons, S., Duerdoth, I.: In: Advances in Nuclear Sciences. Vol. 2, p. 295. New York: Plenum Press (1969)

K. Rashid

B. Fricke

Gesamthochschule Kassel

Universität des Landes Hessen

Fachbereich 18 - Physik

Heinrich-Plett-Straße 40

D-3500 Kassel-Oberzwehren

Federal Republic of Germany 